People prefer simpler content when there are more choices: A time series analysis of lyrical complexity in six decades of American popular music

Michael E. W. Varnum ${ }^{1}$, Jaimie Arona Krems ${ }^{2}$, Colin Morris ${ }^{3}$, Alexandra Wormley ${ }^{1}$, Igor Grossmann ${ }^{4}$

${ }^{1}$ Arizona State University, ${ }^{2}$ Oklahoma State University, ${ }^{3}$ Toronto, Canada, ${ }^{4}$ University of Waterloo

Correspondence to:

${ }^{1}$ Michael Varnum mvarnum@asu.edu

${ }^{2}$ Jaimie Arona Krems

jaimie.krems@okstate.edu

${ }^{3}$ Colin Morris

colin.morris2@gmail.com

${ }^{4}$ Igor Grossmann

igrossma@uwaterloo.ca

Word Count: 4799 Words (excluding references and abstract)

Abstract: 187 Words 


\begin{abstract}
Song lyrics are rich in meaning. In recent years, the lyrical content of popular songs has been used as an index of culture's shifting norms, affect, and values. One particular, recently uncovered, trend is that lyrics of popular songs have become increasingly simple over time. Why? We test the idea that increasing lyrical simplicity is accompanied by a widening array of novel song choices. To do so we examined six decades of popular music in the United States $(N=14,661$ songs $)$. Change in the number of novel song choices predicted change toward greater lyrical simplicity of successful songs. We also examined the cross-temporal relationship between novel song choices and lyrical simplicity using a variety of different techniques to account for the potentially confounding influence of temporal autocorrelation, in each case the relationship held. Finally, this relationship was robust when controlling for a range of critical ecological, socioecological, and cultural factors. The present data provide the first cross-temporal evidence suggesting that real-world cultural transmission may depend on the amount of novel choices in the information landscape.
\end{abstract}




\section{People prefer simpler content when there are more choices: A time series analysis of lyrical complexity in six decades of American popular music}

Music is a human universal (Dissanayacke, 2000; Mehr \& Krasnow, 2017).

Because song — and particularly popular song lyrics — can be so rich in meaning (Cooper 1985; Hayakawa, 1957), social scientists have long explored the ways that such lyrics intersect with some fundamental social processes, including identity formation and person perception (e.g., Hyden \& McCandless, 1983; Marshall \& Naumann, 2018; Reisman, 1957; Rentfrow \& Gosling, 2003, 2006, 2007). More recently, social psychologists have begun to view music as a cultural product and to examine the ways that popular music lyrics reflect important aspects of psychology at the cultural level; the content in popular lyrics indexes changing norms, affect, and values (e.g., Brand, Acerbi, \& Mesoudi, 2019; DeWall, Pond, Campbell, \& Twenge, 2011; Diamond, Bermudez, \& Schensul, 2006; Eastman, Pettijohn, \& Terry, 2015; Lambert et al., 2019; Pettijohn \& Sacco, 2009a, 2009b).

One seemingly remarkable trend in popular music is that the lyrics of successful pop songs have become increasingly simple over time (Morris, 2014). Here, we test one possible explanation for this surprising trend, namely that the amount of novel song choices has increased.

Why might the simplicity or complexity of song lyrics be linked to a song's success? Several lines of evidence suggest that people may have baseline preferences for songs with simpler lyrics. One of the most widely known phenomena in psychology is the mere exposure effect, a phenomenon where repeated exposure to a non-aversive 
stimulus increases preference for it (Bornstein, 1989; Zajonc, 1968, 2001). One implication of this principle for the present question is that simpler, more repetitive lyrics as these pieces essentially have this effect baked into them and thus may tend to be preferred all other things being equal. Further, songs with more repetitive lyrics may enjoy certain advantages in terms of information transmission as they are easier to remember (Margulis, 2014) and likely easier to transmit with fidelity (Bartlett, 1932; Henrich, 2015; Rubin, 1997). Further, recent work has shown that naïve listeners find simpler, more repetitive pieces of music to be more enjoyable, engaging, and memorable (Margulis, 2013, 2014).

Why might more new musical choices increase the success of songs with simpler lyrics? Theory and research from diverse literatures suggest that songs with simpler lyrics might be especially successful when there are more new songs to choose from. First, humans are cognitive misers. People have limited information-processing capacities (e.g., Cowan, 2001), and are known to conserve mental resources (Fiske \& Taylor, 2013). Consequently, humans often use shortcuts in decision-making (Bargh, 1989; Tversky \& Kahneman, 1974). For example, when confronted with the task of evaluating persuasive messages and/or complex decision environments, people are more likely to use heuristics, peripheral cues, and other automatic cognitive processes to evaluate these messages if cognitive resources are limited in some fashion (Eagly \& Chaiken, 1993; Petty \& Cacioppo, 1986). Thus, when there are more products to be evaluated, people may increasingly prefer simpler products as they may require less mental effort to engage with. The mere exposure effect might also have a greater influence on decision making in such contexts as well, given that it too can be thought of as a heuristic or even instinctive 
evaluation. Further, across real-world studies and in-laboratory experiments, when people are confronted with a greater number of options to choose from, they are more likely to choose simpler, less cognitively demanding products (Iyengar \& Kamenica, 2010). Taken together, this work suggests that songs with simpler properties, in this case lyrics, might also be more likely to succeed in times when people are exposed to greater amounts of new songs.

In the present work we test the hypothesis that the trend towards increasingly simple popular music lyrics might accompanied by the increasing number of songs released each year.

\section{Methods}

We gathered cross-temporal data covering a period of six decades (1958-2016) on lyrical compressibility (as an index of simplicity/complexity of song lyrics), amount of novel songs produced (as an index of available novel song choices), and ecological, socioecological, and cultural variables linked to patterns of cultural change in previous research or plausibly related to preference for simple vs. complex aesthetic content.

Lyrical compressibility of successful music. We gathered data from 14,661 songs that entered the Billboard Hot 100 charts spanning the period from 1958 (the charts inception) to 2016. The Billboard Hot 100 tracks the 100 most popular songs each week based on music sales, radio airplay, and internet streaming. To operationalize lyrical complexity (vs. simplicity), we estimated text compressibility. ${ }^{1}$

\footnotetext{
${ }^{1}$ By operationalizing complexity via a compressibility index, we avoided some of the conceptual ambiguity associated with operationalization of complexity in prior research
} 
Compressibility indexes the degree to which song's lyrics have more repetitive and less information dense, and thus simpler, content. We used a variant of the established LZ77 compression algorithm. In brief, the LZ77 algorithm works by finding repeated substrings and replacing them with 'match' objects pointing back to the string's previous occurrence. A match is encoded as a tuple $(D, L)$, with $D$ being the distance to the substring's previous occurrence, and $L$ being its length. We treated these matches as costing 3 bytes. This way, a repeated string only leads to space savings if it is of at least length 4 , and longer repetitions lead to greater relative savings. Given a song $S$, and the set of matches $M$ produced by the LZ77 algorithm when applied to that song, its compressed size is therefore:

$$
\operatorname{compsize}(S)=|S|-\sum_{(D, L) \in M} L-3
$$

Where $|S|$ is the original size of the song's lyrics, measured in characters/bytes. The compression ratios of songs in our dataset (i.e., $|S| /$ compsize $(S)$ ) followed an approximately log-normal distribution, so we operationalized compressibility as the logarithm of this ratio:

(e.g., Jordan, Sterling, Pennebaker, \& Boyd, 2018; Kempe et al., 2004; Rycroft, 2006): Whereas multi-purpose use of a single product may reflect product's complexity from the operational standpoint, it may also represent greater simplicity from the standpoint of consumer psychology. Further, song lyrics are tractable to work with when using an automated compression algorithm. 


$$
\operatorname{compressibility}(S)=\log \left(\frac{|S|}{\operatorname{compsize}(S)}\right)
$$

We computed mean compressibility for each year based on all songs that entered the Hot 100 charts in a given year for which we were able to scrape lyrics (1958-2016).

We used the LZ77 compression algorithm because of its intimate connection to textual repetition. Most of the byte savings when compressing song lyrics arise from large, multi-line sections (most importantly the chorus, and chorus-like hooks). Another significant contributor are multi-word phrases, which may be repeated in variations across different lines for poetic effect (e.g. the anaphoric verses in Lady Gaga's Bad Romance: "I want your ugly / I want your disease / I want your everything..."). The compression may make use of repeated individual words, or even sub-word units that repeat (perhaps incidentally), but their contribution to the overall compressibility is low.

Higher compression scores signify more repetition and therefore higher simplicity. For example, Daft Punk's 1997 song “Around the World” repeats the title 144 times and has a compressibility score of 5.42 (the maximum in this sample). Nat King Cole's "The Christmas Song” (1961) has a low compression score of 0.11.

Novel music production. In the spirit of the multiverse analyses (Steegen et al., 2016), we used three separate indicators to assess the amount of new music to which people are likely exposed in a given year. For each year (1958-2016) we computed the total number of songs which made the Hot100 chart, the number of musical releases per year according to Discogs (Discogs.com), and the number of Wikipedia entries about songs first published or performed each year (Wikipedia.org).

Ecological control variables. Previous work suggests a wide range of cultural changes are linked to key dimensions of the ecology including resource levels, pathogen 
threat, and sources of external threat including climate and armed conflict. Thus we used publicly accessible data on markers of several major ecological threats and affordances in the US that have previously been linked to either patterns of cultural change or cultural variation as control variables (Sng et al., 2018; Varnum \& Grossmann, 2017). These included: GDP per capita, GDP growth, unemployment, pathogen prevalence, climatic stress, and participation of the US in major armed conflicts. Previous work suggests that these variables might potentially affect preference for simplicity in aesthetic products. For example, resource scarcity has been linked to greater conformity (Stephens, Markus, \& Townsend, 2007) and cross-temporal work has found that greater resource levels are linked to more innovation and creative output (Varnum \& Grossmann, 2019) and less conformity (Bianchi, 2016; Grossmann \& Varnum, 2015). Higher levels of infectious disease have also been linked to more conformity (Murray, Trudeau, \& Schaller, 2011; Horita \& Masanori, 2017), traditionalism (Tybur, et al., 2016), and tight social norms (Gelfand, et al., 2011; Jackson, et al., 2019). Finally, external threats, due to climate or war, have been linked to more traditional outlooks and tight social norms (Gelfand, et al., 2011).

The data used in our analyses covered the years 1958-2016. Data on GDP per capita and GDP growth were gathered from macrotrends.net, data on the other markers came from Varnum \& Grossmann (2016) and updates from the original data sources used in that manuscript.

Other socio-ecological control variables. Given the possibility that a preference for simpler English lyrics might be linked to shifts in the amount of people for whom English may not be a first language, we used data on the number of green cards issued 
from the Department of Homeland Security as a marker of immigration. Further, previous work suggests that ethnic fractionalization may increase populations tendencies to exaggerate emotional displays in order to ensure meanings are successfully conveyed across diverse audiences (Rychlowska, et al., 2015; Wood, Rychlowska, \& Neidenthal, 2016). In a similar vein, it might be that ethnic fractionalization also increases preferences for simpler, more repetitive lyrics as such content would be easier to convey and understand to a wide range of audiences, hence we used data on ethnic fractionalization from the US Census Bureau as a control variable as well.

Research on the consequences of residential mobility also suggests that perhaps this variable might also affect lyrical preferences. Previous studies have linked residential mobility to greater susceptibility to the mere exposure effect and greater preference for familiar cultural products (Oishi, Miao, Koo, Kisling, \& Ratliff, 2012). Thus it may be that mobility is also linked to a preference for songs with simpler lyrics. To assess mobility we gathered data on percentage of the US population that changed residence within the US from the US Census Bureau.

It might also be the case that when products that succeed with a larger audience may be ones that are simpler - - what is popularly thought of as appealing to the lowest common denominator. During the time period in question, the US population grew substantially, thus it might be that increasing success of songs with simpler lyrics may reflect growth in audience size. Hence we also gathered data on the total size of the US population from macrotrends.net.

Cultural value control variables. Finally, we gathered data on indicators of conservative ideology and collectivism as such variables might also be plausibly linked to 
preferences for simple vs. complex cultural products. Prior work has found conservatives show a preference for simple and unambiguous art, speech patterns, and literature (Wilson et al., 1973; Schoonvelde et al., 2019; Suedfeld, 2010; McAllister \& Anderson, 1991; Jost et al., 2003 though see also Conway, et al., 2016). We operationalized conservativism as the average percent of annual survey respondents in Gallup polls identifying as conservative.

An orientation toward collectivism vs. individualism has also been linked to cross-cultural differences in aesthetic preferences and expression (Morling, 2016; Nand et al, 2014; Sensaki, Masuda, \& Nand, 2016), thus we opted to include an index of cultural level collectivism as a control variable as well. Here we used Grossmann \& Varnum's (2015) collectivism index based on frequency of collectivism related words in the Google Ngrams American English corpus.

Analytic procedure. Where possible we use non-parametric ordinal-level measures of correlation or partial correlation (Kendall's rank correlation coefficient $\tau$ ), which provides estimate of similarity of the orderings of the data when ranked by each of the quantities. Since Fechner's initial work on time series analyses, Kendall's $\tau$ has been a preferred metric for examining cross-temporal relationships (Kruskal, 1958). It provides a conservative estimate, which is preferred because time series data is rarely normally distributed. Results were comparable when we used Pearson's $r$ or partial Pearson correlations. First, we examined zero-order relationships between each of the three indices of available novel song choices and average lyrical compressibility of popular songs. Next, we created a composite index of novel song choices and assessed the robustness of the hypothesized link between amount of novel song choices and average 
lyrical compressibility of popular songs by controlling for a host of ecological, socioecological, and cultural factors that might plausibly influence cultural level preference for simplicity vs. complexity. Next, we performed a series of corrective analyses, controlling for the possibly spurious nature of the relationship between our key time series due to temporal autocorrelation.

Given the range of possibilities of correcting for temporal autocorrelation, we opted to perform three different types of analyses that correct or account for the possibility that observed relationships might be spurious as a function of autocorrelation in the time series. First, we computed adjusted significance thresholds based on the Tiokhin-Hruschka procedure (Tiokhin \& Hruschka, 2017). Second, we detrended our novel song production and lyrical compressibility time series by residualizing for year and assessed the correlation between our detrended variables. Third, we used an automated auto-regressive integrated moving average forecasting model (auto.ARIMA) to assess the relationship between novel song choices and lyrical compressibility (Hyndman \& Khandakar, 2008). This technique involves a machine learning algorithm that tests a number of different possible models which vary in autoregressive components, differencing, and moving average components, as well as whether they include an exogenous predictor. Additionally, we used auto.ARIMA to generate a forecast for future patterns of lyrical compressibility (2017-2046).

Data availability. All data and reproducible code for analyses reported in the manuscript are available on the Open Science Framework (https://osf.io/qnsmj/). 


\section{Results}

Indicators of novel song choices and average lyrical compressibility. Mean lyrical compressibility (i.e., simplicity) of songs increased over time, Kendall's $\tau=.726$, $p<.001$, as did number of songs making the Hot 100 charts per year, Kendall's $\tau=.425$, $p<.001$, number of music releases according to Discogs per year, Kendall's $\tau=.973, p<$ .001 , and number of Wikipedia entries for songs by year of publication, Kendall's $\tau=$ $.871, p<.001$. Consistent with our predictions, mean lyrical compressibility per year was positively correlated with amount of novel music produced per year as operationalized by three distinct indicators, Kendall's $\tau$ ( $n$ songs in Hot 100 charts/year) $=.429, p<.001$, Kendall's $\tau$ ( $n$ Discogs music releases / year) $=.721, p<.001$, Kendall's $\tau$ ( $n$ Wikipedia entries about songs/year) $=.680, p<.001$.

Analyses of the composite index of novel song choices. Hot100 songs, Discog music releases, and Wikipedia song entries were highly correlated, $.41<$ Kendall's $\tau$ 's $\leq$ .87 , and formed a single principle component with highest loadings by the Wikipedia song entries (.98), and weakest loading by the Hot 100 songs (.88). To avoid multicollinearity, we used component scores for further analyses. Overall, this index of novel music production was strongly positively related to compressibility, Kendall's $\tau=$ $.714, p<.001$.

Robustness analyses: control variables. This PCA-based composite index remained significantly related to lyrical compressibility when controlling separately for each of the 12 specified control variables, $.220<$ partial Kendall's $\tau$ 's $<.770$, p's $<.02$ (see Table 1 for details). Full correlations between these variables are presented in Figure 1. 
Figure 1. Correlations between variables. Values in squares are Kendall's $\tau$ 's. Shaded squares indicate statistically significant correlations at $\mathrm{p}<.05$.

Lyric Compressibility Music Production GDP per Capita GDP Growth Unemployment 0.1 Pathogen Prevalence

Climatic Stress

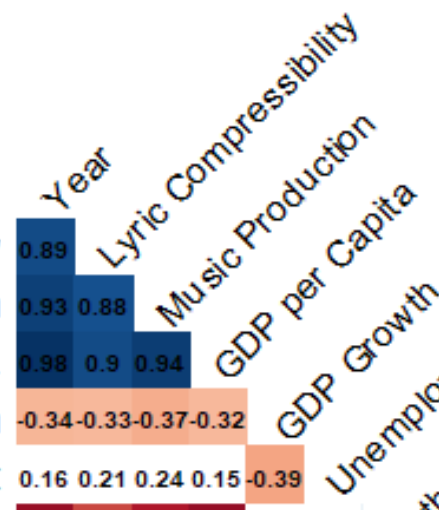

$-0.2-0.13-0.12-0.240 .060 .160 .24$

Major Armed Conflict 0.33 0.31 0.48 0.37-0.17-0.04-0.48-0.05 Immigration

Ethnic Heterogeneity

Residential Mobility

Population Size

Conservatism

Collectivisim
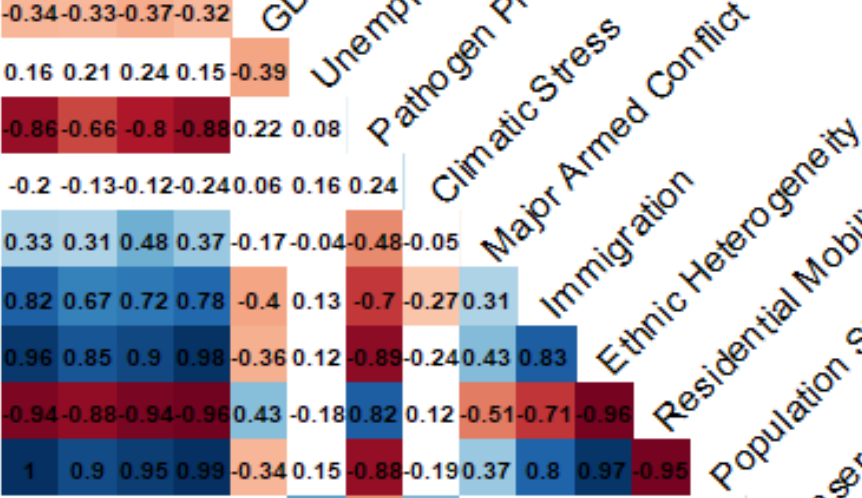

$$
-0.46+1
$$
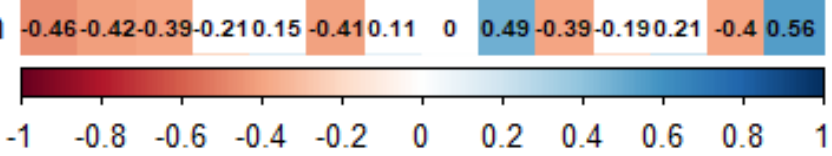
Table 1. Partial correlations between novel music production index and average lyrical compressibility.

\begin{tabular}{|c|c|c|}
\hline Control Variable & $\begin{array}{c}\text { Partial Kendall's } \tau \\
\text { Novel Music Production \& } \\
\text { Lyrical Compressibility }\end{array}$ & p-value \\
\hline $\begin{array}{l}\text { GDP per capita } \\
\text { GDP growth } \\
\text { Unemployment } \\
\text { Pathogen Prevalence } \\
\text { Climatic Stress } \\
\text { Armed Conflict }\end{array}$ & $\begin{array}{l}.245 \\
.695 \\
.753 \\
.596 \\
.710 \\
.696\end{array}$ & $\begin{aligned} & .007 \\
&< .001 \\
&< .001 \\
&< .001 \\
&<.001 \\
&<.001\end{aligned}$ \\
\hline $\begin{array}{l}\text { Immigration } \\
\text { Ethnic Heterogeneity } \\
\text { Residential Mobility } \\
\text { Population Size }\end{array}$ & $\begin{array}{l}.539 \\
.267 \\
.436 \\
.231\end{array}$ & $\begin{array}{r}<.001 \\
.003 \\
<.001 \\
.01\end{array}$ \\
\hline $\begin{array}{l}\text { Conservatism } \\
\text { Collectivism }\end{array}$ & $\begin{array}{l}.670 \\
.610\end{array}$ & $\begin{array}{l}<.001 \\
<.001\end{array}$ \\
\hline
\end{tabular}

Robustness analyses: auto-correlation. Importantly, the correlation between this composite index of novel song choices and average lyrical compressibility remained significant when adjusting significance thresholds using the Tiokhin-Hruschka method to account for observed auto-correlation in the two time series, $r=.877$, corrected $p<.001$. As an alternative method for dealing with autocorrelation, we also detrended the time series by residualizing out the linear impact of year. The correlation for our detrended variables remained significant, Kendall's $\tau=.222, p<.02$.

Given the time series nature of our data, another way to test the hypothesized link between amount of new songs available and average compressibility of these songs can involve an automated ARIMA algorithm (auto.ARIMA) within the forecast package 
(Hyndman, Athanasopoulos, Bergmeir, Caceres, Chhay,...\& Yasmeen, 2020) in in $R$ 4.0.0 (R Core Team, 2020). This machine-learning algorithm inspects the time-series data to fit the optimal forecasting function. The auto-regressive $(A R(p))$ component refers to the use of past values in the regression equation for the series $\mathrm{Y}$. The auto-regressive parameter $\mathrm{p}$ specifies the number of lags used in the model. A moving average $(M A(q))$ component represents the error of the model as a combination of previous error terms $e_{t}$. The order $q$ determines the number of terms to include in the model. ARIMA models are well-suited for long-term time series, such as the historic patterns in the present data. The automated algorithm within the forecast package searches through combinations of order parameters and picks the set that optimizes model fit criteria, comparing Akaike information criteria (AIC) or Bayesian information criteria (BIC) of respective models. Notably, the automated forecasting approach allows us to specify an exogenous predictor such as novel song choices, such that the automated function can evaluate the extent to which this exogenous predictor improves the fit above and beyond the decomposition of the time-series of the dependent variable. In other words, the automated function provides a conservative way to see whether an exogenous predictor such as the novel song choices index improves accuracy in forecasts of the lyrical compressibility. If the final model selected by auto.ARIMA includes our putative exogenous variable (in this case amount of novel song choices), then this suggests that this variable helps the model to achieve optimal fit to the data.

The results of this automated forecasting procedure indicated that a model with a positive autoregressive component, $B=.527, S E=.124$, and a positive contribution of the novel music production index, $B=.059, S E=.008$, provides the best fit to the data: 
$\mathrm{y}_{\mathrm{t}}($ lyrical compressibility function $)=.983+.527 \mathrm{y}_{\mathrm{t}-1}+.059 \mathrm{x}+\mathrm{e}_{\mathrm{t}}$

This model estimation suggests that the index of novel song choices contributes to average lyrical compressibility above and beyond the temporal autocorrelation observed for average lyrical compressibility. Further, the coefficient for the index of novel song choices was statistically significant, $z=6.95, p<.001$.

We also ran an alternative set of auto.ARIMA analyses where we set novel song choices as the dependent variable and average lyrical compressibility as an exogenous predictor. The results of this automated forecasting procedure indicated that a model with two positive moving average components, $B=1.176, S E=.242$, and $B=.487, S E=.164$, and a positive contribution of average lyrical compressibility, $B=5.067, S E=2.207$, provides the best fit to the data:

$\mathrm{y}_{\mathrm{t}}($ novel music production function $)=-4.991+1.176 \varepsilon_{\mathrm{t}-1}+0.487 \varepsilon_{\mathrm{t}-2}+5.067 \mathrm{x}+\mathrm{e}_{\mathrm{t}}$ The coefficient for lyrical compressibility was statistically significant, $\mathrm{z}=2.30, p=.02$. Comparison of the Aikeke Information Criterion (AIC) and Bayesian Information Criterion (BIC) values for our primary and alternative models suggest that our primary model with novel song choices as an exogenous predictor and lyrical compressibility as the dependent variable, $\mathrm{AIC}=-235.84, \mathrm{BIC}=-227.53$, is superior to the alternate model with lyrical compressibility as an exogenous predictor and novel song choices as the dependent variable, $\mathrm{AIC}=58.36, \mathrm{BIC}=68.75$.

Forecasting. As a final step, we generated a forecast for average lyrical compressibility for four decades after the last data point in our time series. This is in keeping with recommendations by Varnum \& Grossmann (2017) that papers analyzing past patterns of cultural change provide forecasts for the future. These forecasts enable a 
test of this theoretical model against concrete future cultural trends. Using the automated ARIMA algorithm, we also identified the best function for the novel song choices data, which we used to estimate the subsequent 40 data points. In turn, we used this estimated data in conjunction with the compressibility function to forecast the further development of lyrical compressibility. Results of this model suggest that lyrical compressibility will continue to increase over the next several decades (see Figure 2).

Figure 2. Novel music production-based forecast for future lyrical compressibility from regression with ARMIA $(1,0,0)$ and index of novel song choices as an exogenous predictor.

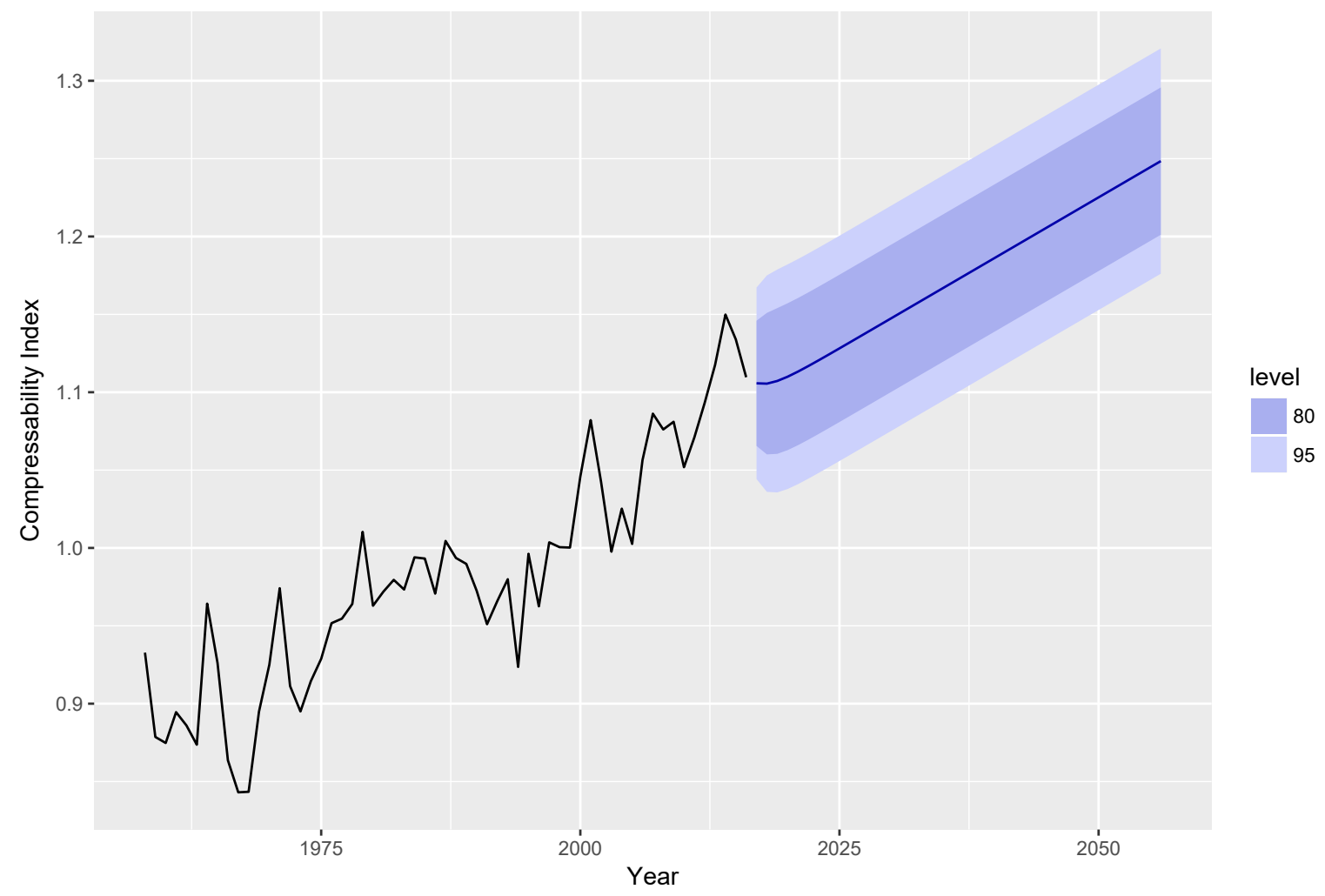




\section{Discussion}

Popular music lyrics have recently been used to inform the cultural transmission of emotional expression (Brand et al., 2019; Schellenberg \& von Scheve, 2012), as an index of culture-level changes in self- versus other-focus (DeWall et al., 2011), and as a reflection of cultural mood in respond to economic and social threats (e.g., Sacco \& Pettijohn, 2009a, 2009b). But one major trend in popular music lyrics remained unexplained: Why are these lyrics becoming increasingly simple over time? Our results were consistent with the hypothesis that increasing novel song choices might lead to greater success for songs with simpler lyrics.

The relationship between mean lyrical compressibility and the amount of novel music produced each year was robust. We observed significant positive associations across three operationalizations of the amount of novel song choices and the average lyrical compressibility of popular songs. Further, the relationship between amount of novel song choices and average compressibility of popular songs remained significant when controlling for a host ecological, socioecological, and cultural factors linked to other types of cultural change. Importantly, a linkage between amount of new music produced and average compressibility of popular songs also held when accounting for temporal autocorrelation using three distinct methods. Thus the results thus suggest that the amount of novel music produced contributes to changes in average lyrical compressibility above and beyond other plausible causes and autoregressive trends in the data.

Thus the present report adds to a growing body of empirical research using timeseries data to test hypotheses about cultural change (for a review see Varnum \& 
Grossmann, 2017). Here we use big data and time series methods to show that increases in the amount of novel songs appear to be linked to the increasing success of songs with simpler lyrics. What does this tell us more broadly about how American culture has changed? It suggests that preference for complexity at the cultural level may be something that shifts over time. To our knowledge this is the first attempt to systematically empirically assess why such cultural level preferences may change.

\section{Limitations and Future Directions}

First and foremost, our analysis was restricted to a single type of cultural product. It might be the case that empirical analysis of other domains might show similar trends and a similar relationship between amount of novel content and success of simpler content, or it may be that different dynamics are observed when considering television shows, videogames, or other types of cultural products. For example, many have argued that television shows have become more complex and intellectually stimulating in the past few decades (the so-called "Golden Age of Television"). However, empirical work examining complexity over time in other types of cultural products, including movies, news broadcasts, print newspapers, novels, and political speech suggests that there is in fact a broad trend toward simpler contents being increasingly preferred, at least when it comes to the language used in these products (Jordan, et al., 2019). It is noteworthy though, that Jordan and colleagues (2019) used a different measure of complexity, in this case use of a specific set of words indicate cognitive complexity, and that they find that the strength of the decline in complexity varies across different types of cultural products. Hence, future research may attempt to conceptually replicate our work by assessing 
compressibility of other types of cultural products over time and whether the success of such products is linked to the number of options or alternatives within that domain.

It is also worth noting that in the present work we assessed the simplicity of lyrics. Songs might be complex or simple in other ways as well, in terms of rhythm, melody, number of instruments played etc. Analyses of these features is beyond the scope of the present work, but it would be interesting to see the extent to which similar or divergent patterns are observed in these facets of successful popular music over time.

Our analysis was also limited to songs that were relatively successful over time, i.e. those that made the Billboard Hot 100 chart. This sample, although quite large $(\mathrm{N}>$ $14,000)$ may not be representative of all songs produced during this period. And it is conceivable that different trends are observed for lyrical complexity overall vs. among songs that are commercially successful and that different forces may affect lyrical complexity among this broader set of cultural products. That said, the aim of the present work was to understand what shapes the success of cultural products over time, rather than to use the broadest possible set of cultural products as a way to gain insight into other phenomena at the population level (see supplement for an extended discussion of this issue).

Our work is also limited by the fact that song success was operationalized by commercial success in the US market. Although some cultural shifts in the past several decades appear to be global in nature, such as rising individualism (Santos, Varnum \& Grossmann, 2017), this need not be the case for all dimensions of culture. Different dynamics may potentially be observed in terms of song success in parts of the world with different values, practices, and ecological conditions. Although such an endeavor is 
beyond the scope of the present manuscript largely due to the lack of equally rich time series data from other countries, it would be worthwhile to try to address this question in the future.

The present work is limited by its correlational nature. Although our findings appeared quite robust across different operationalizations of the independent variable, when accounting for autocorrelation in various ways, and when controlling for a host of plausible ecological and socioecological factors and cultural values which have shifted over time, we cannot completely rule out all alternative explanations for increasing success of songs with simpler lyrics. Future work might attempt to quantify society level time series trends in conformity or other biases linked to lyrical affect and music sampling (e.g., Brand, 2019; Youngblood, 2019), and assess whether the present findings hold when controlling for these variables as well.

It may also be that people consume music in a different fashion now than in the past when music playing devices were less portable. For example, it may have become more common to primarily listen to music while engaged other activities, hence an increasing preference for songs with simpler content. Thus an interesting avenue for future work may attempt to quantify shifts in where, when, and how we consume music and to explore whether such shifts might be linked to increasing success of songs with simpler lyrics as well.

Finally, future work may also use in-lab methods to explore and disentangle the possible causal mechanisms underlying the link between amount of novel song choices and success of songs with simpler lyrics. For example, transmission chain methods (Mesoudi \& Whiten, 2008) could be employed to explore whether participants might find 
simpler lyrics more pleasing and memorable when there is a greater number of other song-snippets competing for attention versus when there is not.

\section{Conclusion}

Why have the lyrics of pop songs become simpler over time? Our findings suggest that the answer may have to do with the proliferation of new songs available to consumers. The present work represents one of the first attempts to use big data and time series methods to quantify temporal shifts in information transmission dynamics at the societal level. Future work may attempt to replicate and extend these findings into other types of complexity and other types of cultural products. 


\section{References}

Bargh, J. A. (1989). Conditional automaticity: Varieties of automatic influence in social perception and cognition. Unintended Thought, 3, 51-69.

Bartlett, F. C. (1932). Remembering: An experimental and social study. Cambridge: Cambridge University Press.

Bianchi, E. C. (2016). American individualism rises and falls with the economy: Crosstemporal evidence that individualism declines when the economy falters. Journal of Personality and Social Psychology, 111(4), 567.

Bornstein, R. F. (1989). Exposure and affect: overview and meta-analysis of research, 1968-1987. Psychological Bulletin, 106(2), 265.

Brand, C. O., Acerbi, A., \& Mesoudi, A. (2019). Cultural evolution of emotional expression in 50 years of song lyrics. Evolutionary Human Sciences, 1, E11.

Chater, N., \& Vitányi, P. (2003). Simplicity: A unifying principle in cognitive science?. Trends in cognitive sciences, 7(1), 19-22.

Conway, L. G., Gornick, L. J., Houck, S. C., Anderson, C., Stockert, J., Sessoms, D., \& McCue, K. (2016). Are Conservatives Really More Simple-Minded than Liberals? The Domain Specificity of Complex Thinking: Ideology and Complexity. Political Psychology, 37(6), 777-798.

Cooper, V. W. (1985). Women in popular music: A quantitative analysis of feminine images over time. Sex roles, 13(9-10), 499-506.

Cowan, N. (2001). Metatheory of storage capacity limits. Behavioral and brain sciences, 24(1), 154-176.

DeWall, C. N., Pond Jr, R. S., Campbell, W. K., \& Twenge, J. M. (2011). Tuning in to psychological change: Linguistic markers of psychological traits and emotions over time in popular US song lyrics. Psychology of Aesthetics, Creativity, and the Arts, 5(3), 200.

Diamond, S., Bermudez, R., \& Schensul, J. (2006). What's the rap about ecstasy? Popular music lyrics and drug trends among American youth. Journal of Adolescent Research, 21(3), 269-298.

Dissanayake, E. (2000). Antecedents of the temporal arts in early mother-infant interaction. The origins of music, 389-410. 
Eagly, A. H., \& Chaiken, S. (1993). The psychology of attitudes. Harcourt Brace Jovanovich College Publishers.

Eastman, J. T., Pettijohn, I. I., \& Terry, F. (2015). Gone country: An investigation of Billboard country songs of the year across social and economic conditions in the United States. Psychology of Popular Media Culture, 4(2), 155.

Ferrer-i-Cancho, R., Hernández-Fernández, A., Lusseau, D., Agoramoorthy, G., Hsu, M. J., \& Semple, S. (2013). Compression as a universal principle of animal behavior. Cognitive Science, 37(8), 1565-1578.

Fiske, S. T., \& Taylor, S. E. (2013). Social cognition: From brains to culture. Sage.

Gelfand, M. J., Raver, J. L., Nishii, L., Leslie, L. M., Lun, J., Lim, B. C., ... \& Aycan, Z. (2011). Differences between tight and loose cultures: A 33-nation study. Science, 332(6033), 1100-1104.

Grossmann, I., \& Varnum, M. E. (2015). Social structure, infectious diseases, disasters, secularism, and cultural change in America. Psychological Science, 26(3), 311324.

Hayakawa, S. I. Popular songs vs. the facts of life. In B. Rosenberg \& D. M. White (Eds.), Mass culture: The popular arts in America. Glencoe, I11.: Free Press, 1957.

Henrich, J. (2015). The secret of our success: how culture is driving human evolution, domesticating our species, and making us smarter. Princeton University Press.

Hobday, M. (1998). Product complexity, innovation and industrial organisation. Research policy, 26(6), 689-710.

Horita, Y., \& Takezawa, M. (2018). Cultural differences in strength of conformity explained through pathogen stress: a statistical test using hierarchical Bayesian estimation. Frontiers in psychology, 9, 1921.

Hyden, C., \& McCandless, N. J. (1983). Men and women as portrayed in the lyrics of contemporary music. Popular Music \& Society, 9(2), 19-26.

Hyndman R, Athanasopoulos G, Bergmeir C, Caceres G, Chhay L, O'Hara-Wild M, Petropoulos F, Razbash S, Wang E, Yasmeen F (2020)._forecast: Forecasting functions for time series and linear models_. R package version $8.12,<\mathrm{URL}$ : http://pkg.robjhyndman.com/forecast>.

Iyengar, S. S., \& Kamenica, E. (2010). Choice proliferation, simplicity seeking, and asset allocation. Journal of Public Economics, 94(7-8), 530-539. 
Jackson, J. C., Gelfand, M., De, S., \& Fox, A. (2019). The loosening of American culture over 200 years is associated with a creativity-order trade-off. Nature human behaviour, 3(3), 244-250.

Johnson, S. (2005). Everything Bad is Good for You: How Popular Culture is Making Us Smarter. Penguin: London.

Jordan, K. N., Sterling, J., Pennebaker, J. W., \& Boyd, R. L. (2019). Examining longterm trends in politics and culture through language of political leaders and cultural institutions. Proceedings of the National Academy of Sciences, 116(9), 3476-3481.

Jost, J. T., Glaser, J., Kruglanski, A. W., \& Sulloway, F. J. (2003). Political conservatism as motivated social cognition. Psychological Bulletin, 129(3), 339.

Lambert, B., Kontonatsios, G., Mauch, M., Kokkoris, T., Jockers, M., Ananiadou, S., \& Leroi, A. M. (2020). The pace of modern culture. Nature Human Behaviour, 1-9.

Margulis, E. H. (2014). Verbatim repetition and musical engagement. Psychomusicology: Music, Mind, and Brain, 24(2), 157.

Margulis, E. H. (2013). Aesthetic responses to repetition in unfamiliar music. Empirical Studies of the Arts, 31(1), 45-57.

Marshall, S. R., \& Naumann, L. P. (2018). What's your favorite music? Music preferences cue racial identity. Journal of Research in Personality, 76, 74-91.

McAllister, P. O., \& Anderson, A. (1991). Conservatism and the comprehension of implausible text. European Journal of Social Psychology, 21(2), 147-164.

Mehr, S. A., \& Krasnow, M. M. (2017). Parent-offspring conflict and the evolution of infant-directed song. Evolution and Human Behavior, 38(5), 674-684.

Mehr, S., Singh, M., York, H., Glowacki, L., \& Krasnow, M. (2017). Song Excerpts. https://osf. io/5x3qv/

Mesoudi, A., \& Whiten, A. (2008). The multiple roles of cultural transmission experiments in understanding human cultural evolution. Philosophical Transactions of the Royal Society B: Biological Sciences, 363(1509), 3489-3501.

Morling, B. (2016). Cultural difference, inside and out. Social and Personality Psychology Compass, 10(12), 693-706.

Morris, C. (2014). Pop music is stuck on repeat (TedXPenn) [Video file]Retrieved from https://www.youtube.com/watch? $\mathrm{v}=\mathrm{tj}$ FwcmHy5M. 
Murray, D. R., Trudeau, R., \& Schaller, M. (2011). On the origins of cultural differences in conformity: Four tests of the pathogen prevalence hypothesis. Personality and Social Psychology Bulletin, 37(3), 318-329.

Nand, K., Masuda, T., Senzaki, S., \& Ishii, K. (2014). Examining cultural drifts in artworks through history and development: cultural comparisons between Japanese and western landscape paintings and drawings. Frontiers in Psychology, 5, 1041.

Norenzayan, A. (2006). Evolution and transmitted culture. Psychological Inquiry, 17(2), 123-128.

North, A. C., Krause, A. E., \& Ritchie, D. (2020). The relationship between pop music and lyrics: A computerized content analysis of the United Kingdom's weekly top five singles, 1999-2013. Psychology of Music, 0305735619896409.

Oishi, S., Kesebir, S., \& Diener, E. (2011). Income inequality and happiness. Psychological Science, 22(9), 1095-1100.

Oishi, S., Miao, F. F., Koo, M., Kisling, J., \& Ratliff, K. A. (2012). Residential mobility breeds familiarity-seeking. Journal of Personality and Social Psychology, 102(1), 149.

Pettijohn, T. F., \& Sacco Jr, D. F. (2009a). The language of lyrics: An analysis of popular Billboard songs across conditions of social and economic threat. Journal of Language and Social Psychology, 28(3), 297-311.

Pettijohn, T. F., \& Sacco Jr, D. F. (2009b). Tough times, meaningful music, mature performers: Popular Billboard songs and performer preferences across social and economic conditions in the USA. Psychology of Music, 37(2), 155-179.

Petty, R. E., \& Cacioppo, J. T. (1986). The elaboration likelihood model of persuasion. In Communication and persuasion (pp. 1-24). Springer New York.

Rubin, D. C. (1997). Memory in oral traditions: The cognitive psychology of epic, ballads, and counting-out rhymes. Oxford: Oxford University Press.

Rasmussen, E. E., \& Densley, R. L. (2017). Girl in a Country Song: Gender Roles and Objectification of Women in Popular Country Music across 1990 to 2014. Sex Roles, 76(3-4), 188-201.

Rentfrow, P. J., \& Gosling, S. D. (2003). The do re mi's of everyday life: the structure and personality correlates of music preferences. Journal of personality and social psychology, 84(6), 1236. 
Rentfrow, P. J., \& Gosling, S. D. (2006). Message in a ballad: The role of music preferences in interpersonal perception. Psychological Science, 17(3), 236-242.

Rentfrow, P. J., \& Gosling, S. D. (2007). The content and validity of music-genre stereotypes among college students. Psychology of music, 35(2), 306-326.

Riesman, D. Listening to popular music. In B. Rosenberg \& D. M. White (Eds.), Mass culture: The popular arts in America. Glencoe, Ill.: Free Press, 1957.

Rychlowska, M., Miyamoto, Y., Matsumoto, D., Hess, U., Gilboa-Schechtman, E., Kamble, S., ... \& Niedenthal, P. M. (2015). Heterogeneity of long-history migration explains cultural differences in reports of emotional expressivity and the functions of smiles. Proceedings of the National Academy of Sciences, 112(19), E2429-E2436.

Santos, H. C., Varnum, M. E., \& Grossmann, I. (2017). Global increases in individualism. Psychological Science, 28(9), 1228-1239.

Schellenberg, E. G., \& von Scheve, C. (2012). Emotional cues in American popular music: Five decades of the Top 40. Psychology of Aesthetics, Creativity, and the Arts, 6(3), 196.

Schoonvelde, M., Brosius, A., Schumacher, G., \& Bakker, B. N. (2019). Liberals lecture, conservatives communicate: Analyzing complexity and ideology in 381,609 political speeches. PLoS ONE, 14(2).

Senzaki, S., Masuda, T., \& Nand, K. (2014). Holistic versus analytic expressions in artworks: Cross-cultural differences and similarities in drawings and collages by Canadian and Japanese school-age children. Journal of Cross-Cultural Psychology, 45(8), 1297-1316.

Sng, O., Neuberg, S. L., Varnum, M. E. W., \& Kenrick, D. T. (2018). The behavioral ecology of cultural psychological variation. Psychological Review.

Stephens, N. M., Markus, H. R., \& Townsend, S. S. (2007). Choice as an act of meaning: the case of social class. Journal of personality and social psychology, 93(5), 814830.

Suedfeld, P. (2010). The Cognitive Processing of Politics and Politicians: Archival Studies of Conceptual and Integrative Complexity. Journal of Personality, 78(6), 1669-1702.

Tamariz, M., \& Kirby, S. (2015). Culture: copying, compression, and conventionality. Cognitive science, 39(1), 171-183. 
Tiokhin, L., \& Hruschka, D. (2017). No evidence that an Ebola outbreak influenced voting preferences in the 2014 elections after controlling for time-series autocorrelation: A Commentary on Beall, Hofer, and Schaller (2016). Psychological Science, 28(9), 1358-1360.

Tybur, J. M., Inbar, Y., Aarøe, L., Barclay, P., Barlow, F. K., De Barra, M., ... \& Consedine, N. S. (2016). Parasite stress and pathogen avoidance relate to distinct dimensions of political ideology across 30 nations. Proceedings of the National Academy of Sciences, 113(44), 12408-12413.

Tversky, A., \& Kahneman, D. (1974). Judgment under uncertainty: Heuristics and biases. Science, 185(4157), 1124-1131.

Twenge, J. M., \& Park, H. (2017). The Decline in Adult Activities Among US Adolescents, 1976-2016. Child Development.

Varnum, M. E. W., \& Grossmann, I. (2016). Pathogen prevalence is associated with cultural changes in gender equality. Nature Human Behaviour, l(1), 003.

Varnum, M. E. W., \& Grossmann, I. (2017). Cultural change: The how and the why. Perspectives on Psychological Science, 12(6), 956-972.

Varnum, M. E. W., \& Grossmann, I. (2019). The wealth -> life history -> innovation account of the industrial revolution is largely inconsistent with empirical time series data. Behavioral and Brain Sciences, 42, e212.

Wilson, G. D., Ausman, J., \& Mathews, T. R. (1973). Conservatism and art preferences. Journal of Personality and Social Psychology, 25(2), 286-288.

Wood, A., Rychlowska, M., \& Niedenthal, P. M. (2016). Heterogeneity of long-history migration predicts emotion recognition accuracy. Emotion, 16(4), 413-420.

Youngblood, M. (2019). Cultural transmission modes of music sampling traditions remain stable despite delocalization in the digital age. PloS one, 14(2).

Zajonc, R. B. (1968). Attitudinal effects of mere exposure. Journal of Personality and Social Psychology, 9(2), 1-27.

Zajonc, R. B. (2001). Mere exposure: A gateway to the subliminal. Current Directions in Psychological Science, 10(6), 224-228. 
